\title{
The behavioral variant of Alzheimer's
} disease does not show a selective loss of Von Economo and phylogenetically related neurons in the anterior cingulate cortex

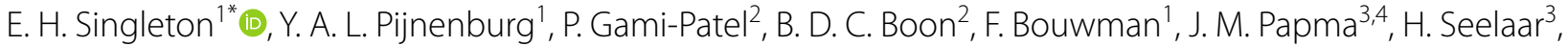 \\ P. Scheltens ${ }^{1}$, L. T. Grinberg ${ }^{5,6}$, S. Spina ${ }^{5}$, A. L. Nana ${ }^{5}$, G. D. Rabinovici, ${ }^{6,7}$, W. W. Seeley ${ }^{5,6}$, R. Ossenkoppele ${ }^{1,8}$ and \\ A. A. Dijkstra ${ }^{2^{*}}$
}

\begin{abstract}
Background: The neurobiological origins of the early and predominant behavioral changes seen in the behavioral variant of Alzheimer's disease (bvAD) remain unclear. A selective loss of Von Economo neurons (VENs) and phylogenetically related neurons have been observed in behavioral variant frontotemporal dementia (bvFTD) and several psychiatric diseases. Here, we assessed whether these specific neuronal populations show a selective loss in bvAD.

Methods: VENs and GABA receptor subunit theta (GABRQ)-immunoreactive pyramidal neurons of the anterior cingulate cortex (ACC) were quantified in post-mortem tissue of patients with bvAD $(n=9)$ and compared to typical AD $(\mathrm{tAD}, n=6)$, bvFTD due to frontotemporal lobar degeneration based on TDP-43 pathology (FTLD, $n=18$ ) and controls $(n=13)$ using ANCOVAs adjusted for age and Bonferroni corrected. In addition, ratios of VENs and GABRQ-immunoreactive (GABRQ-ir) pyramidal neurons over all Layer 5 neurons were compared between groups to correct for overall Layer 5 neuronal loss.

Results: The number of VENs or GABRQ-ir neurons did not differ significantly between bVAD (VENs: 26.0 \pm 15.3 , GABRQ-ir pyramidal: $260.4 \pm 87.1$ ) and tAD (VENs: $32.0 \pm 18.1, p=1.00$, GABRQ-ir pyramidal: $349.8 \pm 109.6, p=0.38$ ) and controls (VENs: $33.5 \pm 20.3, p=1.00$, GABRQ-ir pyramidal: $339.4 \pm 95.9, p=0.37$ ). Compared to bvFTD, patients with bvAD showed significantly more GABRQ-ir pyramidal neurons (bvFTD: $140.5 \pm 82.658, p=0.01$ ) and no significant differences in number of VENs (bvFTD: $10.9 \pm 13.8, p=0.13$ ). Results were similar when assessing the number of VENs and GABRQ-ir relative to all neurons of Layer 5.
\end{abstract}

Discussion: VENs and phylogenetically related neurons did not show a selective loss in the ACC in patients with bvAD. Our results suggest that, unlike in bvFTD, the clinical presentation in bVAD may not be related to the loss of VENs and related neurons in the ACC.

\footnotetext{
*Correspondence: e.singleton@amsterdamumc.nl;

a.dijkstra@amsterdamumc.nl

${ }^{1}$ Alzheimer Center Amsterdam, Department of Neurology, Amsterdam

Neuroscience, Vrije Universiteit Amsterdam, Amsterdam UMC,

Amsterdam, the Netherlands

2 Department of Pathology, Amsterdam Neuroscience, Vrije Universiteit

Amsterdam, Amsterdam UMC, Amsterdam, the Netherlands

Full list of author information is available at the end of the article
}

(c) The Author(s) 2022. Open Access This article is licensed under a Creative Commons Attribution 4.0 International License, which permits use, sharing, adaptation, distribution and reproduction in any medium or format, as long as you give appropriate credit to the original author(s) and the source, provide a link to the Creative Commons licence, and indicate if changes were made. The images or other third party material in this article are included in the article's Creative Commons licence, unless indicated otherwise in a credit line to the material. If material is not included in the article's Creative Commons licence and your intended use is not permitted by statutory regulation or exceeds the permitted use, you will need to obtain permission directly from the copyright holder. To view a copy of this licence, visit http://creativecommons.org/licenses/by/4.0/. The Creative Commons Public Domain Dedication waiver (http://creativeco mmons.org/publicdomain/zero/1.0/) applies to the data made available in this article, unless otherwise stated in a credit line to the data. 
Keywords: Alzheimer's disease, Behavior, Frontotemporal dementia, Pathology, Von Economo Neurons

\section{Introduction}

Von Economo neurons (VENs) constitute a specialized type of large bipolar projection neurons, located in Layer 5 of the anterior cingulate cortex (ACC) and frontoinsular cortex (FI) [1] and have been associated with behavioral dysregulation in bvFTD [2-4], autism [5, 6], and schizophrenia $[7,8]$. These neurons are part of a larger neuronal population in the same cortical layer, the $\mathrm{GABA}_{\mathrm{A}}$ receptor subunit theta (GABRQ) immunoreactive neurons [9]. In addition to VENs, the GABRQ neuronal population in the ACC is also reduced in bvFTD [10] and schizophrenia [11]. The behavioral variant of Alzheimer's disease (bvAD) shows clinical overlap with behavioral variant of frontotemporal dementia (bvFTD), characterized by early and predominantly behavioral changes, such as disinhibition, apathy, and compulsiveness, with $\mathrm{AD}$ as underlying pathology [12-15]. Previous investigations showed conflicting results regarding predominant involvement of anterior regions that are essential for socio-emotional functioning in terms of atrophy [12, 16-19], hypometabolism [20-23], or and tau pathology [19, 24-27]. In the absence of a clear neuroanatomical origin, an alternative explanation may not lie in topographical spreading of $\mathrm{AD}$ pathological processes, but instead in a selective loss of specialized neurons regulating social behavior. A previous study showed no selective loss of VENs in the ACC in bvAD with substantial Lewy body pathology [28], leaving the role of VENs in "pure" bvAD unknown. Furthermore, the phylogenetically related neurons have not been assessed in bvAD. Here, we studied the number of VENs and GABRQ-immunoreactive (GABRQ-ir) neurons in carefully phenotyped bvAD cases compared to patients with typical AD (tAD), bvFTD and controls. We hypothesized that bvAD would show comparable reductions in numbers and ratios of VENs and phylogenetically related neurons with bvFTD, and greater reductions compared to controls and tAD patients.

\section{Methods}

\section{Participants}

Post-mortem brain tissue was obtained from the University of California San Francisco Neurodegenerative Disease Brain Bank (UCSF NDBB) for 3 bvAD cases and the remaining cases were obtained from the Netherlands Brain Bank (NBB; Amsterdam, The Netherlands, https:// www.brainbank.nl). Participants and their caregivers provided informed consent to undergo autopsy according to the Declaration of Helsinki, and all study procedures were approved by the institutional review boards at the participating sites. Extensive clinical reports were available from all donors. Patients with bvAD were included if they showed (a) a primary pathological diagnosis of $\mathrm{AD}$ and (b) met during life $\geq 2$ of 6 core clinical criteria for bvFTD [29], consisting of early and predominant apathy, loss of empathy, disinhibition, compulsive behaviors, hyperorality, and dysexecutive functioning based on patient chart reviews (reviewed by AAD, EHS, RO; see Table S1). All bvAD cases showed 3R/4R tau pathology in advanced Braak stage regions, indicative of AD pathology as primary etiology. We included six donors with a typical amnestic presentation of $\mathrm{AD}(\mathrm{tAD})$ [30] and primary pathological diagnosis of $\mathrm{AD}$ [31] and 18 donors with bvFTD [29] with underlying FTLD-TDP pathology, consisting of 9 cases with a C9orf72 repeat expansion, 5 cases with progranulin mutations and 4 cases with a sporadic origin ( $n=1$ TDP-E, $n=1$ TDP-C, $n=2$ TDP-A), according to FTLD consensus criteria [32] as a reference group. Since the role of VENs is less established in FTLD-tau based on previous studies [2-4], we included FTLD-TDP cases only. In addition, we included 13 cognitively unimpaired individuals (Table 1 and Table S1). Donors were included prospectively, with as much age matching as possible, with inherent differences in age between typical $\mathrm{AD}$ and bvAD and bvFTD cases. Donors with significant concomitant pathology leading to mixed pathological diagnoses were excluded from the study; both bvAD and tAD donors were restricted to early-stage PD Lewy body scores (Braak 0-3, or amygdala only) [33] and limbic-only TDP-43 aggregation [34] (Table S3).

\section{Immunohistochemical procedures}

Immunohistochemical procedures were performed as previously reported [10]. Briefly, sequential $10 \mu \mathrm{m}$ sections of the ACC adjacent to the genu were sampled from the right hemisphere and stained for GABA receptor subunit epsilon (GABRE; 1:1000; HPA045918, Sigma Aldrich, St. Louis, MO) and GABRQ (1:750; HPA002063; Sigma Aldrich) and counterstained with hematoxylin as previously reported [10]. Quantification was performed using the Meander option in Stereoinvestigator, and GABRE staining was used to outline Layer $5 \mathrm{a}$ and GABRQ staining to visualize VENs and related neurons. After delineation of Layer 5, two blinded raters (PGP \& AAD) classified each pyramidal neuron and VEN in Layer 5 based on morphology into four groups: GABRQ-ir VENs, GABRQ-ir pyramidal neurons, GABRQ-negative VENs, and GABRQnegative neurons. Previous work performed by the same raters in a similar sample showed interrater reliability of 
Table 1 Demographic and pathological characteristics of participants by diagnostic group

\begin{tabular}{|c|c|c|c|c|c|}
\hline & bvAD & tAD & bvFTD & Controls & $p$-value ${ }^{\dagger}$ \\
\hline N & 9 & 6 & 18 & 13 & \\
\hline Age at death & $70.1(9.0)$ & $79.7(12.7)$ & $64.8(8.8)$ & $66.8(11.4)$ & 0.68 \\
\hline Sex, no. of females, $\%$ & $5,55.6$ & $2,33.3$ & $10,55.6$ & $6,50.0$ & 0.78 \\
\hline Thal stage, mean & $5.0(0.0)$ & $4.8(0.4)$ & $0.61(0.89)$ & $1.67(1.43)$ & $\begin{array}{l}\text { bvAD }>C N, p<0.001, t A D>C N, p<0.001, \text { bvAD }>\text { bvFTD, } \\
p<0.001, \text { tAD }>\text { bvFTD }, p<0.001\end{array}$ \\
\hline Braak stage, mean & $5.6(0.5)$ & $5.6(0.5)$ & $1.11(1.20)$ & $0.67(0.75)$ & bvAD $>C N, p<0.001, \mathrm{tAD}>\mathrm{CN}, p<0.001, \mathrm{tAD}>\mathrm{bvFTD}, p<0.001$ \\
\hline $\begin{array}{l}\text { LBD } \diamond \text { present in amyg- } \\
\text { dala or brainstem, } \%\end{array}$ & 44.4 & 50 & $\mathrm{n} / \mathrm{a}$ & $\mathrm{n} / \mathrm{a}$ & \\
\hline LATE $\square$ present, \% & 11.1 & 50 & $\mathrm{n} / \mathrm{a}$ & $\mathrm{n} / \mathrm{a}$ & \\
\hline
\end{tabular}

${ }^{\dagger}$ Significant differences in age were assessed by an ANOVA test and differences in sex were assessed with a $\chi^{2}$ test, differences in Thal and Braak stages were assessed with emmeans post hoc tests in $R$ version 4.0.2 [35]

$\checkmark \mathrm{LBD}=$ Lewy body disease, assessed as present or absent

$\square$ LATE = Limbic predominant age related TDP-43 pathology, assessed as present or absent

bvAD behavioral variant of Alzheimer's disease, $t A D$ typical $A D, b v F T D$ behavioral variant frontotemporal dementia

$\alpha=0.06$ (Gami-Patel P, Scarioni M, Bouwman FH, Boon BDC, van Swieten JC, Netherlands Brain Bank, et al: The severity of behavioural symptoms in FTD is linked to the loss of GABRQ-expressing VENs and pyramidal neurons, under review). VENs were distinguished from pyramidal neurons by their large bipolar cell body and thick dendrites [1]. Finally, ratios relative to all Layer 5 neurons were calculated to control for varying size of the ACC and overall neurodegeneration. A step-by-step illustration of these procedures is provided in Fig. 1.

\section{Statistical analyses}

Differences in demographic characteristics were studied using ANOVAs or $\chi^{2}$ tests where appropriate. Main effects in the number of VENs, GABRQ-ir pyramidal neurons, and the ratios VENs/all neurons and GABRQir pyramidal/all neurons were analyzed using ANCOVAs adjusting for age and between group differences were assessed using emmeans post hoc tests, adjusting for age, using a Bonferroni correction in R version 4.0.2 [35].

\section{Results}

\section{Participant characteristics}

Patient characteristics are provided in Table 1, Table S1, and Table S2. Mean age at death was $70.1 \pm 9.0$ in cases with bvAD compared to $79.7 \pm 12.7$ in tAD, $64.8 \pm 8.8$ in bvFTD, and $66.8 \pm 11.4$ in controls; the proportion of females was $55.6 \%$ in bvAD compared to $33.3 \%$ in tAD, $55.6 \%$ in bvFTD, and $50.0 \%$ in controls. There were no significant differences in age and sex among groups $(p=0.68$ and $p=0.78$ resp.).

\section{Copathologies across groups}

Lewy body copathology in the brainstem and/or amygdala was present in $44 \%$ of bvAD and $40 \%$ tAD cases and
TDP-43 copathology in the limbic regions was present in $11 \%$ bvAD and $40 \%$ of tAD cases. In addition, cerebral amyloid angiopathy was present in $89 \%$ of bvAD and $80 \%$ of tAD cases, cerebrovascular disease in $63 \%$ of bvAD and $80 \%$ of typical AD cases and aging-related tau astrogliopathy (ARTAG) was present in $56 \%$ of bvAD and $80 \%$ of typical AD cases.

\section{VENs and GABRQ-immunoreactive neurons across groups} The numbers and ratios of VENs (GABRQ-ir and negative) and GABRQ-ir pyramidal neurons are shown in Fig. 2 and Table S4. After adjustment for age, there was a significant difference between the groups in the number of VENs, $F(1,43)=12.6, p<0.001$, the number of GABRQ-ir pyramidal neurons, $F(1,43)=25.2$, $p<0.0001$, the ratio VENs/all neurons, $F(1,43)=9.0$, $p=0.004$, and the ratio GABRQ-ir pyramidal/all neurons, $F(1,43)=14.9, p<0.001$. Post hoc tests, adjusted for age, showed no significant differences in the number of VENs or GABRQ-ir pyramidal neurons in bvAD (VENs: $26.0 \pm 15.3$, GABRQ-ir pyramidal: $260.4 \pm 87.13$ ) compared to tAD (VENs: $32.0 \pm 18.1, p=1.00$, GABRQir pyramidal: $349.8 \pm 109.6, p=0.38$ ) and controls (VENs: $33.5 \pm 20.3, p=1.00$, GABRQ-ir pyramidal: $339.4 \pm 95.988, p=0.37)$. In order to correct for varying size of the ACC, ratios relative to all Layer 5 neurons of the ACC were analyzed. The ratios of VENs and GABRQir pyramidal vs all neurons showed a similar pattern, with no significant differences between bvAD (ratio VENs/all neurons: $0.009 \pm 0.004$, ratio GABRQ-ir pyramidal/all neurons: $0.098 \pm 0.019$ ) compared to tAD (ratio VENs/ all neurons: $0.012 \pm 0.005, p=1.00$, ratio GABRQ-ir pyramidal/all neurons: $0.133 \pm 0.036, p=0.37$ ) and compared to controls (ratio VENs/all neurons: $0.010 \pm 0.005$, $p=1.00$, ratio GABRQ-ir pyramidal/all neurons: 


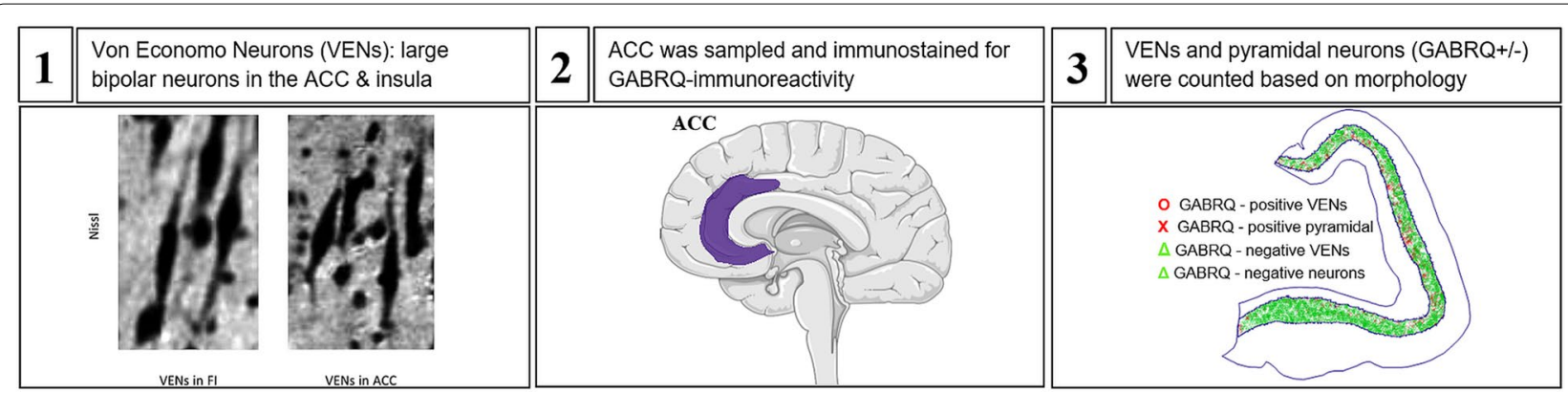

Fig. 1 Schematic overview of methodology. 1 VENs, adapted from Banovac et al. [55]. 2 The location of the ACC in the human cortex. Both images were obtained from smartservier.com. $\mathbf{3}$ The type of neurons in the ACC after staining and counting
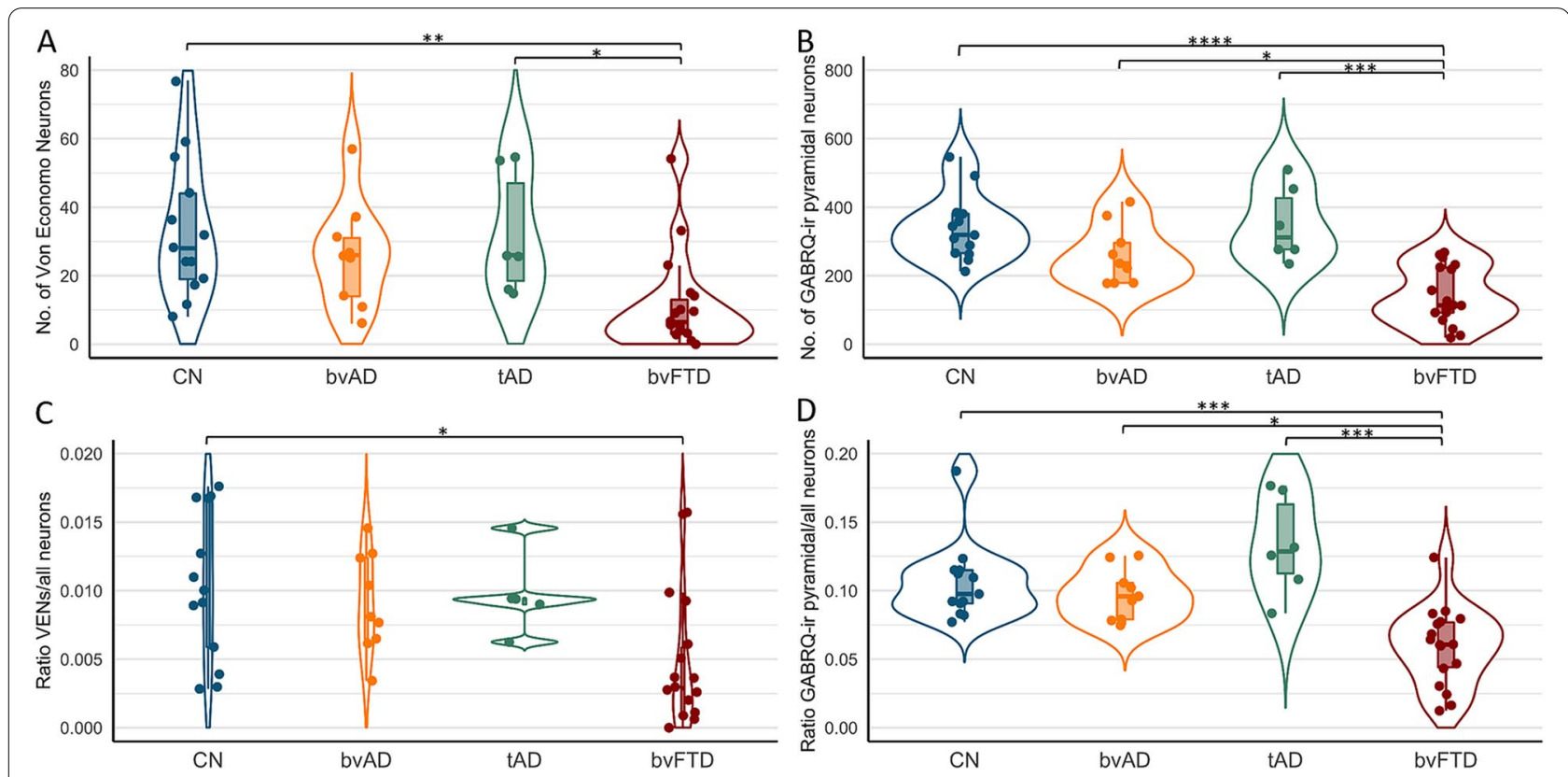

Fig. 2 Numbers and ratios of VENs and GABRQ-immunoreactive pyramidal neurons in bvAD compared to tAD, bvFTD and controls. A The number of VENs counted based on GABRQ-immunohistochemistry across groups, representing the total number of GABRQ-immunopositive VENs and GABRQ-immunonegative VENs, showing no significant differences between bvAD, typical AD, and controls. B The number of GABRQ-immunoreactive pyramidal neurons) across groups, showing no significant differences between bvAD, typical AD, and controls and significantly higher numbers in bVAD and typical AD compared to bvFTD. C The ratio of total VENs (GABRQ-immunopositive and -negative) over all layer 5 neurons in order to correct for effects of neurodegenerative processes, showing no differences between bvAD, typical AD, and controls in turn and significantly lower VENs in bvFTD than controls. D The ratio of GABRQ-immunoreactive pyramidal neurons over all layer 5 neurons, showing no differences between bvAD, typical $A D$ and controls and significantly higher ratios in bVAD and typical AD compared to bvFTD. Results were assessed using emmeans post-hoc tests, adjusting for age, using a Bonferroni correction in R version 4.0.2

$0.106 \pm 0.029, p=1.00)$. As reported before [10], in the current study, FTLD-TDP donors showed lower numbers of VENs (bvFTD: 10.9 \pm 13.8 ), GABRQ-ir pyramidal neurons (bvFTD: $140.439 \pm 82.6)$ and ratios VENs/all neurons (bvFTD: $0.005 \pm 0.005$ ) and GABRQ-ir pyramidal/ all neurons (bvFTD: $0.059 \pm 0.028$ ) compared to controls (VENs, $p=0.003$, GABRQ-ir pyramidal, $p<0.0001$, ratio VENs/all neurons, $p=0.02$, ratio GABRQ-ir pyramidal/ all neurons, $p<0.001$ ) and tAD (VENs, $p=0.03$, GABRQir pyramidal, $p<0.001$, ratio VENs/all neurons: $p=0.07$, ratio GABRQ-ir pyramidal/all neurons: $p<0.001$ ). Here, we show lower though non-significantly different number of VENs (bvFTD: 10.9 $\pm 13.8, p=0.13$ ), GABRQ-ir pyramidal neurons (bvFTD: 150.2 $\pm 91.5, p=0.009$ ), and ratios of VENs/all neurons (bvFTD: $0.005 \pm 0.005$, $p=0.23)$ and significantly lower ratios of GABRQ-ir 
pyramidal/all neurons (bvFTD: $0.063 \pm 0.031, p=0.01$ ) in FTLD-TDP donors than bvAD. Assessment of all subcategories, i.e., the number of GABRQ-ir VENs, GABRQ-negative VENs, GABRQ-ir pyramidal and GABRQ-negative pyramidal are shown in Table S2 and Fig. S1. There was no association between the number of bvFTD symptoms and number of VENs or GABRQ-ir pyramidal neurons in bvAD cases $(p=0.92$ and $p=0.97$ resp., see Fig. S3).

\section{Discussion}

In this study, we examined whether VENs and phylogenetically related neurons in the ACC show a selective loss in bvAD, potentially explaining the early and predominant behavioral changes that characterize this rare atypical variant of $\mathrm{AD}$. Contrary to our hypothesis, we found no selective loss of VENs and GABRQ-ir neurons in bvAD compared to $\mathrm{ADD}$ and controls. These data suggest that, unlike in bvFTD, behavioral alterations in bvAD may not be related to a loss of VENs and phylogenetically related neurons in the ACC.

Our results are in line with a previous study showing no differences in the number of VENs of the ACC between bvAD and tAD [28] in patients with substantial coexisting $\alpha$-synuclein pathology. In a sample of donors without comorbid neocortical $\alpha$-synuclein nor TDP-43 pathology in both bvAD and tAD, our findings confirm the absence of a selective loss of VENs in the ACC in bvAD. Nevertheless, our results suggest a subtle, nonsignificant, trend towards intermediate levels of VENs in bvAD relative to $\mathrm{AD}$ and bvFTD, while the previous study showed relatively higher levels of VENs in bvAD than $\mathrm{tAD}$. In addition, we show no selective loss in bvAD of GABRQ-ir neurons, with significantly higher levels in bvAD compared to bvFTD. The discrepancy between the studies, i.e., the numerically higher densities of VENs in the previous work compared to the intermediate levels in bvAD compared to typical AD and bvFTD observed in our study, might be explained by differences in methods and inclusion of donors with neocortical levels of $\alpha$-synuclein pathology in the work by Tan et al. [28], since neocortical $\alpha$-synuclein can also modulate the behavioral symptoms observed in bvAD. In our cohort, we aimed to include donors with limited copathologies, not reaching the ACC. More likely however, it could be due to the small sample size due to the rarity of this phenotype or due to the heterogeneous clinical and neuropathological presentation of bvAD [36].

Functional properties of VENs have been associated with intuitive decision making [37] in complex situations that are often characteristic of social situations [38]. VENs are located in the ACC and FI, which constitute key regions of the salience network [39] and regulate higher order social emotions like guilt, embarrassment, and empathy as well as the subjective experience of pain and the need for autonomic activity [40], acting as a gateway between soma and psyche [41]. bvFTD shows specific vulnerability in these regions [42], leading to a wide range of social cognition deficits [43, 44]. Although social cognition deficits have been suggested in bvAD [45-48], reports are mainly based on case studies and possibly biased by cognitive deficits in bvAD. Therefore, future studies should incorporate larger groups and apply more objective measures of socioemotional functioning, such as biometric approaches [49] in order to capture the experiential component of social cognition. Based on the lack of loss of VENs and related neurons in the ACC and a lower involvement of the salience network in bvAD than in bvFTD [22], social cognition patterns may show milder deficits in bvAD. This corresponds to the generally similar but milder behavioral profile in bvAD compared to bvFTD (Table S1) [15]. Alternatively, VENs and related neurons may be more likely to show a selective loss in the frontoinsula in bvAD than in the ACC, as subthe frontoinsular hypometabolism was found previously [50], and no differences between bvAD and typical AD were observed in tau pathology within the ACC [26]. In addition, this study did not assess fork cells, which are also related to VENs and selectively targeted in bvFTD [2]. Future studies should explore these hypotheses.

Our finding that there is no reduction of VENs and phylogenetically related neurons in the ACC, combined with predominantly $\mathrm{AD}$-typical patterns of neurodegeneration $[12,19,20,22]$, and the lack of consistent regional difference in tau pathology between bvAD and tAD [24, 26, 51] may suggest more diffuse neurobiological mechanisms to underlie the behavioral alterations in bvAD. Indeed, anterior default mode network [22], micro-level dysregulations, such as amygdalar overactivity [22], may contribute to its phenotype. Alternatively these patients may show developmental or premorbid personality structures that predispose individuals to exacerbation of vulnerable personality features with accumulating AD pathology. Therefore, future studies should aim to better characterize individuals with bvAD neurobiologically in terms of functional networks as well as clinically in terms of personality, coping styles, early life events, and premorbid psychiatric conditions. Alternatively, bvAD may represent a heterogeneous phenotype, in which some patients may show neurobiological features similar to bvFTD and others may show a more "typical AD" neurobiological profile .

\section{Limitations}

The current study should be evaluated in light of its limitations. First, the small sample size due to the rarity of the disease may have hampered statistical power. 
Second, copathologies are common in AD. Although we included donors with low levels of $\alpha$-synuclein and TDP43 copathologies in bvAD and tAD, the potential contribution of these pathologies to their clinical manifestation is currently unknown. CAA and CVD were prevalent in both bvAD and tAD in our sample, which corresponds to recent work showing substantial CAA and vascular lesions in both early- and late-onset $\mathrm{AD}$ cases [52], indicating a certain generalizability of our cohort. Third, the present study selectively sampled the ACC, while VENs and related neurons are also located in high numbers in the FI. Indeed, bvAD showed frontoinsular involvement based on patterns of atrophy $[16,18]$ and hypometabolism [22], while the ACC showed no differences in postmortem tau pathology in bvAD compared to tAD [26]. Alternatively, early preferential tau aggregation within VENs in the ACC could affect behavior, as has been observed in specific MAPT mutations leading to bvFTD [53]. In AD dementia, rare tau aggregations were identified in VENs, whose density was inversely correlated with Braak stage [54]. Future studies should investigate VENs in the FI and include early post-mortem cases in order to study early local aggregation of tau pathology within the ACC and FI in bvAD cases. Fourth, VENs and pyramidal neurons may not express GABRQ-immmunoreactivity, while actually being present, resulting in "phenotypic silencing." In our data, we found no significant differences between groups in the number of GABRQ-negative VENs, GABRQ-negative pyramidal neurons or the percentage of GABRQ-negative VENs of all VENs (all $p>0.05$, Table S5 and Fig. S1). In previous work, VENs were positively correlated with GABRQ-expressing neurons [10], suggesting that the loss of GABRQ-expressing neurons will be similar across groups. However, the possibility of unequal phenotypic silencing across groups cannot be ruled out.

\section{Conclusion}

In conclusion, our results demonstrate no selective loss of VENs and related neurons in the ACC in bvAD. Future research is needed to further elucidate the neurobiological mechanisms constituting the early and predominant behavioral dysregulation in bvAD.

\section{Supplementary Information}

The online version contains supplementary material available at https://doi. org/10.1186/s13195-021-00947-9.

Additional file 1.

\section{Acknowledgements}

The authors would like to acknowledge Evi Berendrecht for her help in conducting the analyses of the current manuscript.

\section{Authors' contributions}

EHS: study design, data analysis, writing manuscript, YALP: study design, intellectual contribution to and critical assessment of manuscript, PGP: data collection and critical appraisal of manuscript, BDCB, JMP, HS, PS, LTG, SS, ANL, GDR, and WW: critical appraisal of manuscript, RO\&AAD; study design, intellectual contribution to and critical assessment and supervision of manuscript. The authors read and approved the final manuscript.

\section{Funding}

Work at the Alzheimer Center Amsterdam was supported by the Netherlands Organisation for Health Research and Development, ZonMw (70-73305-98-1214 to Rik Ossenkoppele, PI). Research of the Alzheimer center Amsterdam is part of the neurodegeneration research program of Amsterdam Neuroscience. The Alzheimer Center Amsterdam is supported by Stichting Alzheimer Nederland and Stichting VUmc fonds. Work at the University of California San Francisco was supported by the NIH National Institute on Aging (NIA) grants R01-AG045611 (to G.D.R.) as well as funding for Aging and Dementia Research Center (NIA P30-AG062422) and PPG (NIA P01-AG019724). In addition, work at UCSF is funded by K24AG053435 to LTG and KO8 AG052648 to SS.

\section{Availability of data and materials}

Data is available upon reasonable request.

\section{Declarations}

Ethical approval and consent to participate

The ethics committees of the Amsterdam UMC - Location VUmc and the Netherlands Brain Bank and the ethic committee of the University of California and Neurodegenerative Disease Brain Bank provided approval for the conduction of this work and all donors gave consent to participate in research.

\section{Publication history}

This manuscript was previously published as a pre-print on the medRxiv website; MS ID\#: MEDRXIV/2021/265649.

\section{Consent for publication}

All donors gave consent for publication of results based on their data.

\section{Competing interests}

Gil D. Rabinovici reports research support from Avid Radiopharmaceuticals, GE Healthcare, Eli Lilly, Life Molecular Imaging; Scientific advisory boards for Axon Neurosciences, Eiasi, Merck, Roche; Associate Editor for JAMA Neurology. Frederik Barkhof reports research support from GE Healthcare, Biogen, Novartis and TEVA; Scientific advisory boards for Roche, Biogen, Merck, Roche. Lundbeck and IXICO. Philip Scheltens serves/has served on the advisory boards of: Genentech, Novartis, Pfizer, Roche, Danone, Nutricia, Jansen Al, Baxter, and Lundbeck. He has been a speaker at symposia organized by Lundbeck, Lilly, Merz, Pfizer, Jansen Al, Danone, Novartis, Roche, and Genentech. He serves on the editorial board of Alzheimer's Research \& Therapy and Alzheimer Disease and Associated Disorders and is a member of the scientific advisory board of the EU Joint Programming Initiative and the French National Plan Alzheimer. The Alzheimer Center receives unrestricted funding from various sources through the VUmc Fonds. Dr. Scheltens receives no personal compensation for the activities mentioned above. Rik Ossenkoppele is an associate editor for Alzheimer's Research \& Therapy.

\section{Author details}

${ }^{1}$ Alzheimer Center Amsterdam, Department of Neurology, Amsterdam Neuroscience, Vrije Universiteit Amsterdam, Amsterdam UMC, Amsterdam, the Netherlands. ${ }^{2}$ Department of Pathology, Amsterdam Neuroscience, Vrije Universiteit Amsterdam, Amsterdam UMC, Amsterdam, the Netherlands.

${ }^{3}$ Neurology, Erasmus University Medical Center, Rotterdam, the Netherlands. ${ }^{4}$ Radiology, Erasmus University Medical Center, Rotterdam, the Netherlands. ${ }^{5}$ Departments of Pathology, University of California San Francisco, San Francisco, USA. ${ }^{6}$ Departments of Neurology, University of California San Francisco, San Francisco, USA. ${ }^{7}$ Radiology and Biomedical Imaging, University of California San Francisco, San Francisco, USA. ${ }^{8}$ Clinical Memory Research Unit, Department of Clinical Sciences Malmö, Lund University, Lund, Sweden. 
Received: 3 November 2021 Accepted: 13 December 2021

Published online: 20 January 2022

\section{References}

1. Economo, C.v., Eine neue Art Spezialzellen des Lobus cinguli und Lobus insulae. Zeitschrift für die gesamte Neurologie und Psychiatrie, 1926. 100(1): p. 706-712.

2. Kim E-J, et al. Selective frontoinsular von Economo neuron and fork cell loss in early behavioral variant frontotemporal dementia. Cerebral Cortex. 2011;22(2):251-9.

3. Santillo AF, Nilsson C, Englund E. von Economo neurones are selectively targeted in frontotemporal dementia. Neuropathol Appl Neurobiol. 2013;39(5):572-9.

4. Seeley WW, et al. Early frontotemporal dementia targets neurons unique to apes and humans. Ann Neurol. 2006;60(6):660-7.

5. Santos M, et al. von Economo neurons in autism: a stereologic study of the frontoinsular cortex in children. Brain Res. 2011;1380:206-17.

6. Simms ML, et al. The anterior cingulate cortex in autism: heterogeneity of qualitative and quantitative cytoarchitectonic features suggests possible subgroups. Acta Neuropathol. 2009;1 18(5):673-84.

7. Brüne $\mathrm{M}$, et al. Von Economo neuron density in the anterior cingulate cortex is reduced in early onset schizophrenia. Acta Neuropathol. 2010;119(6):771-8.

8. Krause M, Theiss C, Brüne M. Ultrastructural alterations of von Economo neurons in the anterior cingulate cortex in schizophrenia. Anat Rec. 2017;300(11):2017-24.

9. Dijkstra AA, et al. Von Economo neurons and fork cells: a neurochemical signature linked to monoaminergic function. Cerebral Cortex. 2016:28(1):131-44.

10. Gami-Patel P, et al. Von Economo neurons are part of a larger neuronal population that are selectively vulnerable in C9orf72 frontotemporal dementia. Neuropathol Appl Neurobiol. 2019;45(7):671-80.

11. Dijkstra AA, et al. Reduction of GABA subunit theta-containing cortical neurons in schizophrenia. Schizophr Res. 2020.

12. Ossenkoppele R, et al. The behavioural/dysexecutive variant of Alzheimer's disease: clinical, neuroimaging and pathological features. Brain. 2015;138(Pt 9):2732-49.

13. Dubois B, et al. Advancing research diagnostic criteria for Alzheimer's disease: the IWG-2 criteria. Lancet Neurol. 2014;13(6):614-29.

14. Graff-Radford J, et al. New insights into atypical Alzheimer's disease in the era of biomarkers. Lancet Neurol. 2021;20(3):222-34.

15. Ossenkoppele R, Singleton EH, Groot C, Dijkstra AA, Eikelboom WS, Seeley WW, Pijnenburg YA. Research criteria for the behavioral variant of Alzheimer disease: a systematic review and meta-analysis. JAMA Neurol. 2021. https://doi.org/10.1001/jamaneurol.2021.4417.

16. Perry DC, et al. Clinicopathological correlations in behavioural variant frontotemporal dementia. Brain. 2017;140(12):3329-45.

17. Phillips JS, et al. Neocortical origin and progression of gray matter atrophy in nonamnestic Alzheimer's disease. Neurobiol Aging. 2018;63:75-87.

18. Phillips JS, et al. Longitudinal progression of grey matter atrophy in nonamnestic Alzheimer's disease. Brain. 2019:142(6):1701-22.

19. Therriault J, et al. Topographical distribution of amyloid- $\beta$, tau and atrophy in behavioral/dysexecutive AD patients. Neurology. 2020. https://doi. org/10.1212/WNL.0000000000011081

20. Bergeron D, et al. Posterior cingulate cortex hypometabolism in nonamnestic variants of Alzheimer's disease. Journal of Alzheimers Disease. 2020;77(4):1569-77.

21. Sala A, et al. Brain metabolic signatures across the Alzheimer's disease spectrum. Eur J Nucl Med Mol Imaging. 2020;47(2):256-69.

22. Singleton $\mathrm{EH}$, et al. Investigating the clinico-anatomical dissociation in the behavioral variant of Alzheimer disease. Alzheimers Res Ther. 2020:12(1):148.

23. Woodward MC, et al. Differentiating the frontal presentation of Alzheimer's disease with FDG-PET. J Alzheimers Dis. 2015:44(1):233-42.

24. Blennerhassett R, et al. Distribution of pathology in frontal variant Alzheimer's disease. J Alzheimers Dis. 2014:39(1):63-70

25. Kawakatsu S, Kobayashi R, Hayashi H. Typical and atypical appearance of early-onset Alzheimer's disease: a clinical, neuroimaging and neuropathological study. Neuropathology. 2017;37(2):150-73.
26. Singleton $\mathrm{E}$, et al. Heterogeneous distribution of tau pathology in the behavioural variant of Alzheimer's disease. J Neurol Neurosurg Psychiatry. 2021; jnnp-2020-325497.

27. Taylor Kl, et al. Clinical course of neuropathologically confirmed frontalvariant Alzheimer's disease. Nat Clin Pract Neurol. 2008:4(4):226-32.

28. Tan RH, et al. Von Economo neurons in behavioral variant frontotemporal dementia with underlying Alzheimer's disease. J Alzheimers Dis. 2019;69(4):963-7.

29. Rascovsky K, et al. Sensitivity of revised diagnostic criteria for the behavioural variant of frontotemporal dementia. Brain. 2011;134(Pt 9):2456-77.

30. McKhann GM, et al. The diagnosis of dementia due to Alzheimer's disease: recommendations from the National Institute on Aging-Alzheimer's Association workgroups on diagnostic guidelines for Alzheimer's disease. Alzheimers Dement. 2011;7(3):263-9.

31. Montine TJ, et al. Multisite assessment of NIA-AA guidelines for the neuropathologic evaluation of Alzheimer's disease. Alzheimer's \& Dementia. 2016;12(2):164-9.

32. Mackenzie IRA, et al. Nomenclature and nosology for neuropathologic subtypes of frontotemporal lobar degeneration: an update. Acta Neuropathol. 2009;119(1):1.

33. Braak H, Braak E. Neuropathological stageing of Alzheimer-related changes. Acta Neuropathol. 1991;82(4):239-59.

34. Nelson PT, et al. Limbic-predominant age-related TDP-43 encephalopathy (LATE): consensus working group report. Brain. 2019;142(6):1503-27.

35. Team, R.C.. R: A language and environment for statistical com-puting. R Foundation for Statistical Computing, Vienna, Austria. URL https://www. R-project. org, 2017.

36. Singleton $\mathrm{EH}$, et al. Heterogeneous distribution of tau pathology in the behavioral variant of Alzheimer's disease. medRxiv. 2020:2020.09.18.20188276.

37. Allman JM, et al. Intuition and autism: a possible role for Von Economo neurons. Trends Cogn Sci. 2005;9(8):367-73.

38. Pauc R, Young A. The history of von Economo neurons (VENs) and their possible role in neurodevelopmental/neuropsychiatric disorders: a literature review. Clinical Chiropractic. 2009;12(3):101-8.

39. Seeley WW, et al. Dissociable intrinsic connectivity networks for salience processing and executive control. J Neurosci. 2007;27(9):2349-56.

40. Allman JM, et al. The von Economo neurons in frontoinsular and anterior cingulate cortex in great apes and humans. Brain Struct Funct. 2010;214(5):495-517.

41. Sorg C, Schlegel J, Förstl H. Von Economo neurons and insular cortex - the bridge between soma and psyche. Neuropsychiatrie : Klinik, Diagnostik, Therapie und Rehabilitation : Organ der Gesellschaft Osterreichischer Nervenarzte und Psychiater. 2010;24(1):64-6.

42. Zhou J, et al. Divergent network connectivity changes in behavioural variant frontotemporal dementia and Alzheimer's disease. Brain. 2010;133(Pt 5):1352-67.

43. Dickerson BC. Dysfunction of social cognition and behavior. Continuum (Minneap Minn). 2015;21 (3 Behavioral Neurology and Neuropsychiatry):660-77.

44. Harciarek M, Cosentino S. Language, executive function and social cognition in the diagnosis of frontotemporal dementia syndromes. Int Rev Psychiatry. 2013;25(2):178-96.

45. de Souza $L C$, et al. Frontal presentation of Alzheimer's disease: a series of patients with biological evidence by CSF biomarkers. Dement Neuropsychol. 2013;7(1):66-74

46. de Souza LC, et al. Behavioral variant of frontotemporal dementia or frontal variant of Alzheimer's disease? A case study. Dement Neuropsychol. 2019:13(3):356-60.

47. Duclos $\mathrm{H}$, et al. Social cognition in the frontal variant of Alzheimer's disease: a case study. J Alzheimers Dis. 2017:55(2):459-63.

48. Wong $\mathrm{S}$, et al. Frontal variant of Alzheimer's disease masquerading as behavioural-variant frontotemporal dementia: a case study comparison. Neurocase. 2019;25(1-2):48-58.

49. Mauss IB, Robinson MD. Measures of emotion: a review. Cognition and Emotion. 2009;23(2):209-37.

50. Singleton $\mathrm{EH}$, et al. Investigating the clinico-anatomical dissociation in the behavioral variant of Alzheimer's disease. medRxiv. 2019:19006676.

51. Balasa M, et al. Clinical features and APOE genotype of pathologically proven early-onset Alzheimer disease. Neurology. 2011;76(20):1720-5. 
52. Spina $\mathrm{S}$, et al. Comorbid neuropathological diagnoses in early versus lateonset Alzheimer's disease. Brain. 2021;144(7):2186-98.

53. Lin LC, et al. Preferential tau aggregation in von Economo neurons and fork cells in frontotemporal lobar degeneration with specific MAPT variants. Acta Neuropathol Commun. 2019;7(1):159.

54. Gefen T, et al. Von Economo neurons of the anterior cingulate across the lifespan and in Alzheimer's disease. Cortex. 2018:99:69-77.

55. Banovac l, et al. Von Economo neurons - primate-specific or commonplace in the mammalian brain? Front Neural Circuits. 2021;15(89).

\section{Publisher's Note}

Springer Nature remains neutral with regard to jurisdictional claims in published maps and institutional affiliations.

- fast, convenient online submission

- thorough peer review by experienced researchers in your field

- rapid publication on acceptance

- support for research data, including large and complex data types

- gold Open Access which fosters wider collaboration and increased citations

- maximum visibility for your research: over $100 \mathrm{M}$ website views per year

At BMC, research is always in progress.

Learn more biomedcentral.com/submissions 